\title{
Static and dynamic properties of 1-kink skyrmion in $\mathrm{Pt} / \mathrm{Co} / \mathrm{MgO}$ trilayer
}

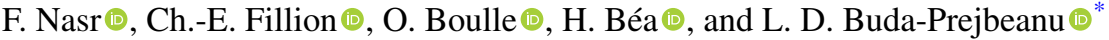 \\ Univ. Grenoble Alpes, CEA, CNRS, Grenoble-INP, SPINTEC, 38000 Grenoble, France
}

(Received 14 June 2021; revised 10 September 2021; accepted 9 November 2021; published 29 November 2021)

The 1-kink skyrmion is a topological spin texture composed of a skyrmion having a chiral kink inside its Néel domain wall, this chiral kink being also called domain wall skyrmion. By micromagnetic simulations we have studied the stability of the 1-kink skyrmion as well as its spin-driven dynamics in $\mathrm{Pt} / \mathrm{Co} / \mathrm{MgO}$. Such trilayer system allows simultaneously the stabilization of various spin textures including skyrmion (Sk, topological charge $Q=1)$, 1-kink skyrmion ( $1 \mathrm{kSk}, Q=2$ ), and domain wall skyrmion (DWSk, $Q=1)$. The 1-kink skyrmion is found to be robust against both thermal fluctuations and normally distributed anisotropy for different grainlike region sizes. Such magnetization pattern has a certain resilience to the variation of the Dzyaloshinskii-Moriya interaction and applied magnetic field. The static analysis is complemented with the investigation of the dynamic regime of motion under electrical current. In the limit of moderate injected current the skyrmion and the 1-kink skyrmion behave similarly. However, depending on the geometry of the injected current, the 1-kink skyrmion can be transformed into a single skyrmion or splits in two skyrmions, opening the path to conceive high density logical devices, enabling novel functionalities for logic operations.

DOI: 10.1103/PhysRevB.104.174441

\section{INTRODUCTION}

Topological nontrivial whirling spin textures such as magnetic skyrmions [1] have recently triggered a lot of interest by virtue of their potential nanosized solitonic nature, stability, and tunability. All these attributes, if combined with the efficient current-induced motion via spin-orbit (SO) torques, make them attractive to build elementary units of digital information [2,3] or to implement neuromorphic computing [4-6]. In a perpendicularly magnetized heavy-metal/ferromagnet/insulator (HM/FM/I) trilayer, such as $\mathrm{Pt} / \mathrm{FeCoB} / \mathrm{MgO}$ or $\mathrm{Pt} / \mathrm{Co} / \mathrm{MgO}$, the chirality of spin arrangements is ascribable to an antisymmetric exchange, interfacial Dzyaloshinskii-Moriya interaction (iDMI) [7-9]. Skyrmions in thin films are nanometer axisymmetric magnetization configurations in which the direction of the central spin is opposite to the direction on its edges. When crossing them radially, the magnetization rotates by $2 \pi$ in a plane perpendicular to the film. Magnetization thus maps once all possible orientation directions, which is quantified by a topological charge $Q=1$, with $Q$ defined as [10,11]:

$$
Q=\frac{1}{4 \pi} \iint d x d y \mathbf{m} \cdot\left(\frac{\partial \mathbf{m}}{\partial x} \times \frac{\partial \mathbf{m}}{\partial y}\right)
$$

where $\mathbf{m}$ is the unitary magnetization vector. Among the possible topological magnetic quasiparticles, which can be stabilized in such peculiar trilayer systems, a lower dimension (1D) nontrivial magnetic texture, named domain wall skyrmion (DWSk), has recently been predicted [12] and experimentally observed using Lorentz transmission electron microscopy (LTEM) [13]. The DWSk is a 1D topological

*Corresponding author: liliana.buda@cea.fr excitation sharing the same integer topological charge as conventional $2 \mathrm{D}$ skyrmions $(Q=1)$. It consists of a localized $2 \pi$ rotation of the in-plane (IP) component of magnetization within a Néel domain wall which stands between two opposite perpendicularly magnetized domains. It can be intuitively viewed as the counterpart of a $2 \pi$-vertical Bloch line coming about in Bloch instead of Néel domain walls. In order to minimize iDMI energy, the domain wall at the location of DWSk is bent [12], thus leading to a so called chiral kink [14]. Skyrmions with chiral kinks have also been predicted as a soliton solution by Kuchkin et al. [14] but the dipolar energy that may stabilize them was neglected. By micromagnetic simulations it was found that, considering dipolar energy, such DWSk may appear inside the domain wall of a skyrmion, but only as a transient state before the stabilization of the skyrmion [15]. Recently, a novel hybrid magnetic texture with nonuniform helicity, the heart-shaped (HS) skyrmion, resulting from two types of DMI contributions, has been acknowledged as a potential candidate to address the skyrmion Hall effect (SkHE) in racetrack memories [16]. Here, we show by micromagnetic simulations using MuMax3 [17] that a DWSk within the domain wall of a skyrmion may be stable in the $\mathrm{Pt} / \mathrm{Co}(0.9 \mathrm{~nm}) / \mathrm{MgO}$ system. The resulting $(1+2)$-dimensional spin texture maps twice the possible orientations of magnetization, which corresponds to $Q=2$. Our simulations show that this spin texture, named hereafter 1-kink skyrmion (1kSk), is stable under thermal fluctuations, granularity, magnetic field, and iDMI variations. We further show that an electrical current, flowing in the plane of the film and inducing spin-transfer torque (STT) or spin-orbit torque (SOT), may displace this $Q=2$ topological charge in a track and even transform it into two $Q=1$ charges (two single skyrmions) in the case of SOT, thus opening perspectives for high density skyrmionic devices. We also predict that inserting 


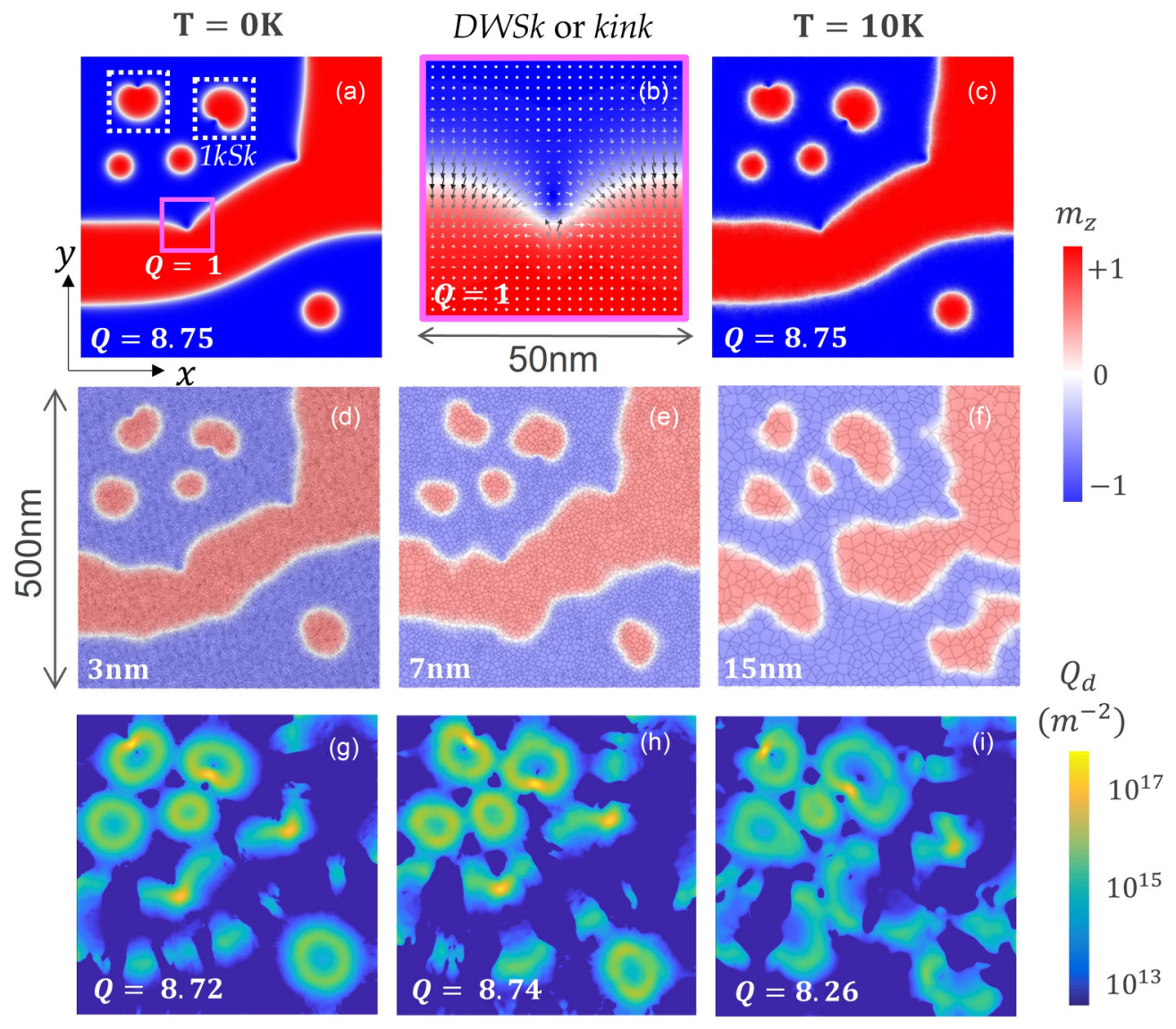

FIG. 1. (a) Multidomain equilibrium state of $0.5 \mu \mathrm{m} \times 0.5 \mu \mathrm{m}$ ideal $\mathrm{Pt} / \mathrm{Co}(0.9 \mathrm{~nm}) / \mathrm{MgO}$ sample at $0 \mathrm{~K}$ under no applied magnetic field. The color scale is associated to the out-of-plane magnetization component $m_{z}$. (b) Internal structure of the left-handed Néel domain wall presenting a kink [pink square in (a)]. (c) Magnetization distribution at $10 \mathrm{~K}$ after $20 \mathrm{~ns}$ of integration remains equivalent. (d)-(f) Evolution of the magnetization distribution at $0 \mathrm{~K}$ upon varying the size of the grains of Co layer from (d) $3 \mathrm{~nm}$ to (e) $7 \mathrm{~nm}$ and to (f) $15 \mathrm{~nm}$ average diameter, with the associated topological charge density in (g)-(i). The total integrated charge is given in the figures. In (d)-(f) the grain distribution is superimposed in gray to the magnetization distribution.

such magnetic stack as the free layer of a magnetic tunnel junction with a perpendicular polarizer layer could allow creating these 1-kink skyrmions by injecting a current and further induce their spinning at tuneable $\mathrm{MHz}-\mathrm{GHz}$ frequencies. The expressions "spin texture" and "soliton" will be used interchangeably to address either the DWSk (also called kink) or the whole 1-kink skyrmion, superposition of a DWSk (or kink) and a Néel-type skyrmion.

\section{NONTRIVIAL SPIN TEXTURES}

The trilayer structure $\mathrm{Pt} / \mathrm{Co} / \mathrm{MgO}$ has been intensively studied in the past, both experimentally and by modeling, since this system allows us to stabilize nanometer scale chiral bubbles at room temperature [18]. The conventional way to stabilize and manipulate these bubbles combines applied magnetic field and/or spin polarized current [19]. In contrast, in our study we explore thermally demagnetized states such as that induced by heating using a laser pulse $[20,21]$. Such disorder offers the system the possibility to reach states inaccessible by simply sweeping the field or injecting in-plane spin-polarized current, due to high energy barriers. Common values for the material parameters of $\mathrm{Pt} / \mathrm{Co}(0.9 \mathrm{~nm}) / \mathrm{MgO}$ sample have been chosen [19]: satura- tion magnetization $M_{s}=1.42 \mathrm{MA} / \mathrm{m}$, uniaxial perpendicular anisotropy constant $K_{u}=1.36 \mathrm{MJ} / \mathrm{m}^{3}$, iDMI constant $D=$ $1.27 / \mathrm{m}^{2}$ (left-handed chirality convention), exchange stiffness $A=16 \mathrm{pJ} / \mathrm{m}$, and damping parameter $\alpha=0.37$. For an accurate description of the magnetization distribution, the sample has been discretized in the film plane with mesh size of $0.488 \mathrm{~nm}$ and $0.9 \mathrm{~nm}$ upon the thickness, respectively.

Figure 1(a) shows a typical equilibrium multidomain state stabilized at zero temperature and under zero applied magnetic field starting from an initial state with random magnetization. We note the simultaneous existence of several features. Firstly, as expected from our previous studies on the same sample [19], there are three stable left-handed chiral bubbles (skyrmions) with perfect circular symmetry. Secondly, one can notice the existence of two left-handed Néel domain walls, one presenting two kinks. The internal structure of such kinks, one being surrounded by a pink square line, is shown in Fig. 1(b): It reveals the existence of a narrow region of the magnetic domain wall where the magnetization points against the chiral direction imposed by the iDM interaction. By following the domain wall, the magnetization is in fact rotating by $2 \pi$ in the plane. Such structures have been numerically predicted and experimentally observed by LTEM in $\mathrm{Pt} / \mathrm{Co} / \mathrm{Ni} / \mathrm{Ir}$ multilayers [13]. They have a topological charge 

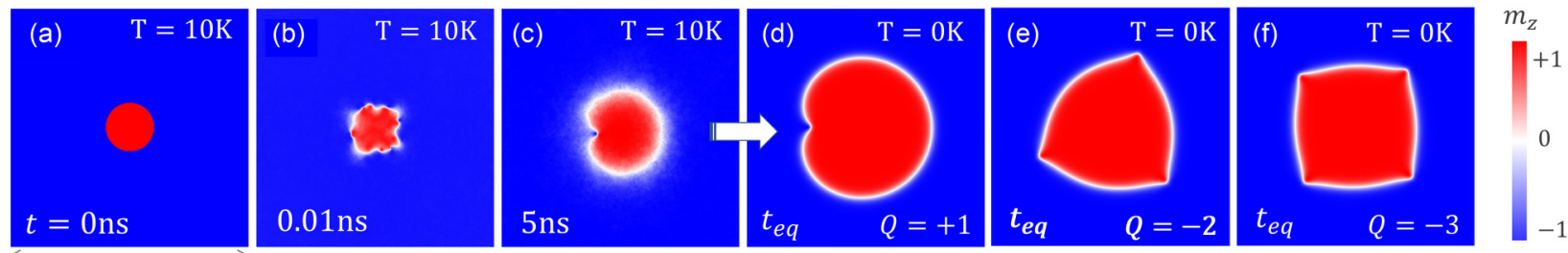

$500 \mathrm{~nm}$

FIG. 2. Thermal driven nucleation of 1-kink skyrmion in $500 \mathrm{~nm}$ wide square platelet: (a) initial state; (b), (c) transient states at $10 \mathrm{~K}$; (d) equilibrium state at $0 \mathrm{~K}$. Same kind of simulation ending up with an equilibrium state with (e) three kinks and (f) four kinks, respectively.

of $Q=1$, as indicated in Fig. 1(b), similarly to a conventional 2D skyrmion. These nontrivial twists of magnetization are located inside the Néel walls and are usually named domain wall skyrmion (DWSk). Finally and interestingly, there are also two 1-kinked chiral bubbles, labeled hereafter as 1-kink skyrmions (1kSk), associated to the presence of a DWSk inserted in their circular domain wall, highlighted by a white dashed square in Fig. 1(a) and carrying a topological charge of $Q=2$. The static and dynamic properties of these $1 \mathrm{kSk}$ are discussed in the following sections.

Both DWSk and 1kSk are found to be stable and resilient against small thermal fluctuations $(T=10 \mathrm{~K}$, see Appendix for details), as shown in Fig. 1(c). Furthermore, the kinks are indicative that the competition between various internal interactions might lead to magnetic configurations tolerating some degree of frustration. The kink inside a Néel domain wall generates a typical deformation of the domain wall surface and gives a cardioidlike shape to the bubble that might be seen as a fingerprint of the chiral kink. Moreover, experimentally, the observations by various imaging techniques have shown that the domain wall surfaces in $\mathrm{Pt} / \mathrm{Co} / \mathrm{MgO}$ are not smooth but with many deformations [22]. Nevertheless these deformations have been interpreted until now as due exclusively to the pinning centers because of internal granular structure of the Co layer. To study the resilience of the kinks against the defects, distributions of grains are generated with different sizes. Particularly, the modeling of sputtered polycrystalline materials, composed of many crystallites of variable size and orientation, is accomplished by discretizing the homogeneous samples into grainlike regions, using 2D Voronoi tessellation. A normal distribution of magnetic anisotropy throughout the defects is defined, with standard deviation to mean value set to $10 \%$. Also, to account for the existence of grain boundaries, the exchange stiffness between two adjacent grains is reduced by $10 \%$. For completeness, three cases were treated with increasing grain size, i.e., $3 \mathrm{~nm}, 7 \mathrm{~nm}$, and $15 \mathrm{~nm}$, respectively. The stable state of the ideal sample [Fig. 1(a)] was relaxed in the granular samples [Figs. 1(d)-1(f)]. We observe that the surface of the domain walls accommodates with the grain topography, as anticipated, but the initial twist of the magnetization inside the kinks is preserved. The impact of the grains becomes stronger upon increasing the crystallite size; some kinks undergo fragmentation while rearranging according to the energy landscape, jointly with a major modification of the magnetization pattern. However, in the limit of moderate disorder (pinning), the kinks are not annihilated and techniques such as LTEM should be able to detect and/or differentiate them from pinning driven distortions. The kinks are corresponding to very steep variation in space of the magnetization and, similarly to the conventional skyrmions, they are characterized by an integer topological charge $Q=1$ [Fig. 1(b)]. Figures 1(g)-1(i) present the distribution of the topological charge density $Q_{d}$ in the three cases with grains. Several very small areas with a high $Q_{d}$ are located in the kink regions (yellow color). The length scale of the kinks is very short (typical size of $30 \mathrm{~nm}$ ), as the zoom in Fig. 1(b) reveals. As a consequence, their accurate description calls for a very fine discretization of the space. Using a mesh size above $1 \mathrm{~nm}$ did not allow us to access and correctly describe the strong gradient of the magnetization at the level of the kinks, and the magnetization relaxed to stable states with much smoother structures. This observation is in line with the previous studies of vertical Bloch lines or Bloch points, seen as very special spin textures [23-25]. The 1-kink skyrmion is even more intriguing since its $(1+2)$-dimensional structure results from the insertion of a very small DWSk (kink), i.e., a 1D skyrmion, inside the domain wall of a conventional and larger 2D skyrmion.

\section{1-KINK SKYRMION THERMAL NUCLEATION}

Random distributions as those induced by heat transfer due an ultrafast laser pulse may stimulate the generation and stabilization of multiple kinks after magnetization recovery process [Fig. 1(a)]. The role played by a randomly distributed thermal field in the nucleation of DWSk is also evidenced in the following numerical experiment depicted in Fig. 2. The initial magnetization pattern of Fig. 2(a) shows a circular magnetic domain antiparallel to the magnetic environment, usually adopted to numerically stabilize and further characterize a conventional skyrmion. With no thermal field (see Appendix for definition), during the energy relaxation transient phase, a Néel-type domain wall of well-defined chirality would be generated, in such a way of minimizing both symmetric (smoother magnetization gradient) and antisymmetric (homochirality) exchange interaction energies. This process would end up with a single 2D Néel skyrmion. By contrast, having an external random perturbation during this stage, e.g., induced by a stochastic thermal field, excites the nucleation of extremely confined kinks ( $Q=+1$, concave profile) and antikinks $(Q=-1$, convex profile) in such domain wall, as Fig. 2(b) shows. Kinks and antikinks undergo pair annihilation. This process, with the right balance between them $\left(N_{\text {kink }}=N_{a \text {-kink }}+1\right)$, may end up with a 1-kink skyrmion [Fig. 2(c)], i.e., a unique kink in the domain wall, which survives thermal field removal [Fig. 2(d)]. As shown Figs. 2(e) 

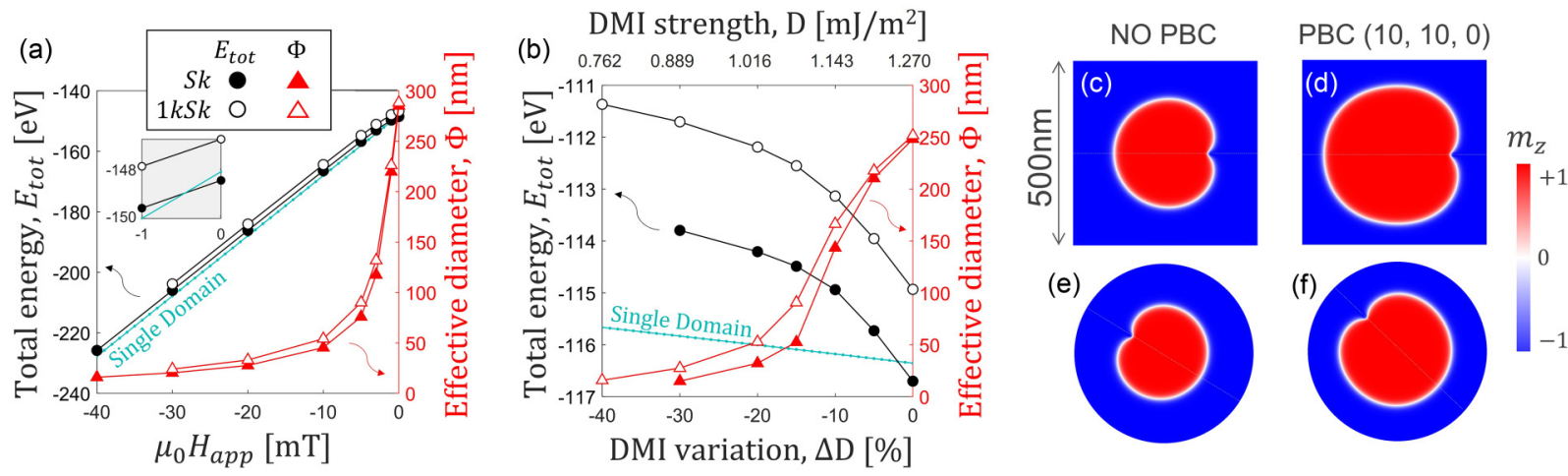

FIG. 3. Dependence of the total energy and of the effective diameter on (a) the out-of-plane magnetic field (square geometry) and (b) on the iDMI variation (circular geometry and no applied magnetic field). The blue lines in (a) and (b) indicate the total energy for the single domain state. From (c) to (f): impact of dipolar field on 1-kink skyrmion size and orientation. In detail, $0 \mathrm{~K}$ out-of-plane equilibrium magnetization distribution with no applied magnetic field for (c), (d) square geometry and (e), (f) circular geometry, without (c), (e) and with (d), (f) periodic boundary conditions, respectively.

and 2(f) this procedure does not allow a precise control on the number of kinks, since the thermal nucleation process is stochastic.

In other words, the heat energy, either due to the ambient temperature (during relaxation) or to a laser pulse (prior to relaxation), assists the magnetization to locally counteract the iDM interaction, bringing out local magnetic textures, which break the chiral homogeneity of the domain wall. This ultimately proves that domain wall skyrmions not only withstand thermally induced fluctuation but are effectively triggered by it. Against this backdrop, the dipolar field seems to play an important role, promoting the local whirling of the in-plane magnetization (to loop the magnetic field flux lines radiated by the domain wall), as the magnetic configuration thermally fluctuates. To corroborate this idea, the dipolar interaction was numerically disabled and no twists of magnetization, i.e., no future DWSk, were in fact observed in the domain wall during the transient time.

\section{1-KINK SKYRMION STATIC PROPERTIES}

The size of the 1-kink skyrmion can be modulated both by external control parameters such as the applied magnetic field and internal material parameters such as the strength of the iDMI. Its effective diameter is evaluated from the total area of the $1 \mathrm{kSk} d_{\mathrm{eff}}=\sqrt{4 S / \pi}$, with $S$ its area. Figures 3(a) and 3(b) summarize the impact of the out-of-plane magnetic field as well as that of the iDMI on the size and the energy of the $1 \mathrm{kSk}$ and compare it to the conventional 2D skyrmion. Upon reinforcing the applied magnetic field pointing against the core (red magnetization region), the $1 \mathrm{kSk}$ size shrinks rapidly [Fig. 3(a)] in a similar manner as the skyrmion but with a slightly larger size. Between $\mu_{0} H_{\text {app }}=-40 \mathrm{mT}$ and $-30 \mathrm{mT}$ the $1 \mathrm{kSk}$ undergoes such forceful compression that its domain wall loses the elasticity requested to stabilize the kink: The $1 \mathrm{kSk}$ converts into a single skyrmion which is then stable for higher field amplitudes. It appears that the three stable states in our sample of $\mathrm{Pt} / \mathrm{Co}(0.9 \mathrm{~nm}) / \mathrm{MgO}$, namely single skyrmion, 1-kink skyrmion, and single domain, are very close in energy [Fig. 3(a)]. Even if the $1 \mathrm{kSk}$ energy remains slightly larger $(\sim 1 \%)$ than that of the skyrmion, one can notice that the difference with the single domain is also relatively small. The single domain state appears to be the fundamental state in the negative range of Zeeman field. However, when the external magnetic field is removed, the optimal tradeoff between the dipolar and the symmetric and antisymmetric exchange interactions make the single skyrmion the ground state of the sample.

A similar contraction of the $1 \mathrm{kSk}$, observed for negative magnetic field values, occurs when the iDMI coefficient is reduced with respect to the initial iDMI value of $1.27 / \mathrm{m}^{2}$ [Fig. 3(b)]. Reasonably, this is verified by the increase of the total energy, due to the cost of the Néel domain wall which is less reduced by the iDMI. In that case, the $1 \mathrm{kSk}$ size decrease is accompanied by a reduction of the kink curvature due to iDMI decrease [12], which costs less elastic energy in the domain wall. This makes $1 \mathrm{kSk}$ strongly resilient as it remains stable up to a decrease of $\Delta D=-50 \%$. Surprisingly, conventional skyrmion is annihilated for smaller iDMI reduction around $\Delta D=-40 \%$, likely due to the smaller topological charge, that would provide a weaker topological protection in this defect-free sample at $0 \mathrm{~K}$.

The finite size and the geometry of the sample are also impacting the $1 \mathrm{kSk}$ behavior. Indeed, by varying the sample shape, the activation or deactivation of periodic boundary conditions (PBC) becomes a control parameter to tune the isotropicity of the magnetic environment surrounding the topological soliton. This allows us to investigate the impact of the stray field radiated by the magnetic background on the space orientation of the kink as well as on the effective diameter. For the PBC implementation, MuMax3 adopts a macro geometry approach [17]. In detail, $\mathrm{PBC}\left(N_{x}, N_{y}, N_{z}\right)$ introduces a periodic repetition of the simulation domain, with the dipolar field taken from the $N_{i}$ next neighboring samples along $x, y$, and $z$ directions. In the present work, the periodicity is just set to be in the plane $\left(N_{x}=N_{y} \neq 0, N_{z}=0\right)$. We used $\left(N_{x}=N_{y}=10\right)$ for our sample since we have observed a saturation of the effect of dipolar field after this value.

Figure 3(c) shows how, in case of square geometry and no $\mathrm{PBC}$, the anisotropicity of the magnetic field flux lines drives the local kink to align along either the $x$ or $y$ axis, which are energetically equivalent by symmetry. This is obtained 
independently on the initial kink orientation. For conventional skyrmions, the cylindrical symmetry leads to isotropic texture. On the contrary, the $1 \mathrm{kSk}$ manifests a radial dependence of its domain wall position, consequence of the local bending of the domain wall. Upon this, the $1 \mathrm{kSk}$ shortest axis at the kink position lines up with the direction along which the extension of the outer magnetic domain is minimized as well. This may be understood as a way to minimize dipolar energy as this orientation optimizes alternating up and down domains along all in-plane directions. Specifically, along a given in-plane direction, a 1D domain can be regarded as a cascade of pinpoint magnetic dipoles, either aligned along $+z$ (up domain) or $-z$ (down domain), each one defined in a unit cell of the discrete space. In this regard, the optimal $1 \mathrm{kSk}$ configuration is that providing the best compensation between the number of $u p$ and down magnetic dipoles along all in-plane directions, thus minimizing dipolar energy which results from their mutual interaction. The application of PBC induces an expansion of the magnetic background in the immediate surrounding of the topological soliton that is consequently dilated, as shown in Fig. 3(d). The kink orientation in this case is also along $x$ or $y$. This may be explained by the fact that in each surrounding cell there is also a $1 \mathrm{kSk}$ (periodicity of magnetic configuration), thus the kink aligns along the direction of the other $1 \mathrm{kSk}$ so that there is the larger distance between neighboring solitons, thanks to the bending of the domain wall. This allows the $1 \mathrm{kSk}$ cores to be further away from each other and thus to minimize dipolar energy cost. This behavior is thus similar to the case of the isolated pillar (without PBC).

Overall, the same characteristic behavior is observed for circular shape, in which case the inherent geometrical isotropicity of the sample is disrupted by the application of PBC because of the identical copies contacting the magnetic background along the $x$ and $y$ axis. Figure 3(e) illustrates the $1 \mathrm{kSk}$ random orientation in the absence of $\mathrm{PBC}$, attesting the energy equivalence of all angular positions. On the contrary, as illustrated in Fig. 3(f), when PBC are imposed, the $1 \mathrm{kSk}$, beyond expanding, reorients itself to align the kink along one of the isoenergetic axis minimizing the dipolar field, now matching the main diagonals of the square. This can be explained by considering the void between one disk and its first neighbor: The smallest number of up magnetic dipoles, found along the $1 \mathrm{kSk}$ shortest axis, is optimally compensated along the main diagonal of the squared simulation window, due to the missing magnetic material, viewable as a lack of down magnetic dipoles.

\section{MOTION OF 1-KINK SKYRMION IN TRACKS UNDER CURRENT}

The static proprieties of 1-kink skyrmion appear to be similar to that of the conventional skyrmion and the presence of the chiral kink induces relative small changes. Hereafter we summarize the behavior of the 1-kink skyrmion under a current injection in the film plane, generating either spin-transfer torque (STT) or spin-orbit torque (SOT). The details about the STT and SOT models are given in the Appendix.

In the case of spin-transfer torque [Figs. 4(a), 4(b), and $4(\mathrm{e})$ ], we observe that the $1 \mathrm{kSk}$ presents a motion similar to that of the single skyrmion. The $1 \mathrm{kSk}$ moves along the electron flow with an additional skyrmion Hall angle (SkHA) [26-29], slightly smaller than for the conventional skyrmion [see Fig. 4(a) vs Fig. 4(b)]. The effective diameter of both spin textures behaves similarly during the current induced motion. The velocity of the $1 \mathrm{kSk}$ is slightly larger, by $1.8 \%$ on average, than for the skyrmion [Fig. 4(e)]. The main difference is the reorientation of the kink in order to drive the $1 \mathrm{kSk}$ motion, which is of course not observed in the single skyrmion due to its cylindrical symmetry (see Supplementary Video SV1). This kink orientation might be explained by the fact that the STT in the DWSk is large as magnetization varies rapidly in space inside the kink. These results are in line with the chiral droplet reaction to STT [30].

In the next part we have submitted the single and 1-kink skyrmions to SOT [31] [see Figs. 4(c), 4(d), and 4(f)]. In the regime of current densities below $500 \times 10^{9} \mathrm{~A} / \mathrm{m}^{2}$, the $1 \mathrm{kSk}$ velocity is smaller than the single skyrmion by $40 \%$ on average. The $1 \mathrm{kSk}$ motion presents a slightly larger skyrmion Hall angle with respect to the single skyrmion. To better understand the reason underneath this behavior, the analytical SkHA was estimated according to the following equation [32]:

$$
\Theta_{\mathrm{SkH}}=\frac{G}{\alpha \mathcal{D}}
$$

where $\alpha$ is the Gilbert damping coefficient. Particularly, the terms $G$ and $\mathcal{D}$ were computed by implementing the following analytical models for the dissipative matrix $\mathcal{D}$ and the gyromagnetic vector $\mathcal{G}=G \hat{\mathbf{z}}$ [26]:

$$
\begin{aligned}
G & =-\frac{M_{s} t}{\gamma} \iint d x d y \mathbf{m} \cdot\left(\frac{\partial \mathbf{m}}{\partial x}\right) \times\left(\frac{\partial \mathbf{m}}{\partial y}\right) \\
\mathcal{D} & =\left(\begin{array}{ll}
\mathcal{D}_{x x} & \mathcal{D}_{x y} \\
\mathcal{D}_{y x} & \mathcal{D}_{y y}
\end{array}\right), \mathcal{D}_{i j}=\frac{M_{s} t}{\gamma} \iint d x d y\left(\frac{\partial \mathbf{m}}{\partial x_{i}} \cdot \frac{\partial \mathbf{m}}{\partial x_{j}}\right)
\end{aligned}
$$

where $i, j$ stands for $x$ or $y, M_{s}$ is the saturation magnetization, $t$ is the thickness of the FM, and $\gamma$ is the gyromagnetic ratio.

First, as expected, the analysis of numerical results in the steady state regime shows that $G^{1 \mathrm{kSk}}=2 G^{\mathrm{Sk}}$, implying that the simplified expression $G=-\left(M_{s} t / \gamma\right) \cdot 4 \pi Q$, valid for conventional skyrmion, can also be adopted for $1 \mathrm{kSk}$. Secondly, again consistently with the magnetic skyrmion description, the main diagonal elements of the dissipative tensor, computed in the steady-state regime, turned out to be nearly equivalent $\left(\mathcal{D}_{x x}=\mathcal{D}_{y y}=\mathcal{D}\right)$, and several orders of magnitude larger than $\mathcal{D}_{x y}$ and $\mathcal{D}_{y x}$, which can thus be neglected. Also, it was found that the $1 \mathrm{kSk}$ topological charge $Q=2$, responsible for the doubled $G$ value, is not fully compensated by $\mathcal{D}$, being $\mathcal{D}^{1 \mathrm{kSk}} \sim 1.9 \mathcal{D}^{\mathrm{Sk}}$. This finally explains the slightly larger SkHA measured for the $1 \mathrm{kSk}$. Finally, the orientation of the kink during motion is different than when driven by STT. In the case of SOT, the kink orients nearly perpendicular to the motion direction. This may be understood due to the nonzero (net) transverse component of the driving force (with respect to the applied current), with a strong local action in correspondence with the kink. As a matter of fact, for a purely Néel (respectively Bloch) magnetic skyrmion, the damping like (DL) SOT driving force, is collinear (respectively transverse) to the current density vector. As visible in Fig. 4(d), the kink is slightly distorted under SOT, the bottom part being 


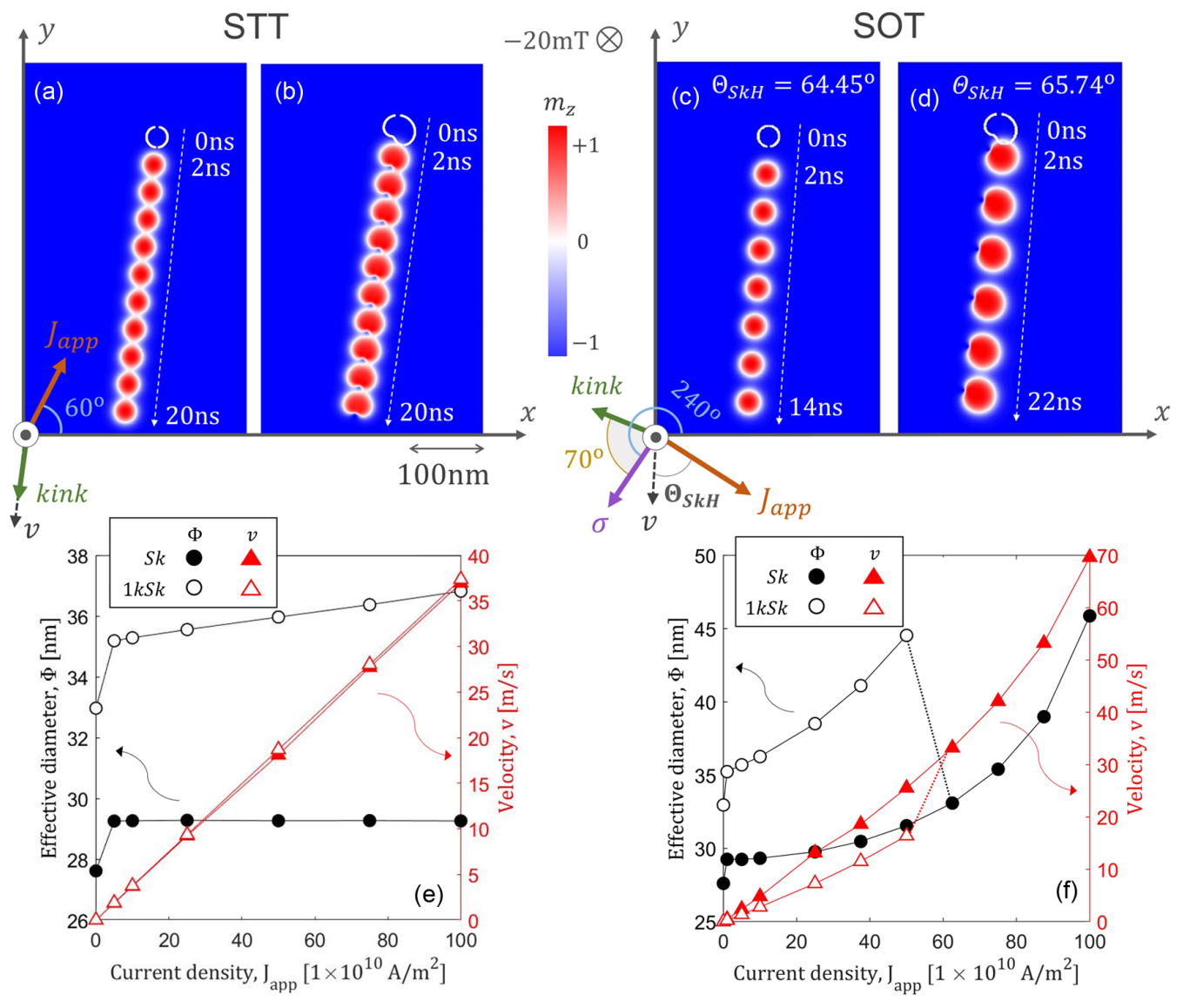

FIG. 4. Comparison of motion of skyrmion (a), (c) and 1-kink skyrmion (b), (d) under STT with electrical current polarization 100\% (a), (b) and SOT (c), (d). The position of skyrmion or 1-kink skyrmion before current application in (a)-(d) is given by their contour in white line. The successive images correspond to $2 \mathrm{~ns}$ integration time steps. Applied current direction is given by the brown arrow, its magnitude is $J_{\text {app }}=500 \times 10^{9} \mathrm{~A} / \mathrm{m}^{2}$, and the spin polarization is $10 \%$. Applied magnetic field along $\hat{z}$ is $\mu_{0} H_{\text {app }}=-20 \mathrm{mT}$. For SOT case, the injected spin polarization $\sigma$, perpendicular to current, is given by the purple arrow in (c). For both STT and SOT, the orientation of velocity and of the 1-kink skyrmion are represented as black and green arrows, respectively. (e), (f) Plots of effective diameter and velocity of skyrmion (full symbols) and 1-kink skyrmion (empty symbols) as a function of current density for STT (e) and SOT (f) cases. For SOT, currents higher than $500 \times 10^{9} \mathrm{~A} / \mathrm{m}^{2}$ lead to the transformation of the 1-kink skyrmion into one or two single skyrmions (see Fig. 5).

a bit more bent than the top part, making one of the two Bloch-like sides of opposite chirality prevailing. Upon this statement, one can infer that, due to this noncompensation of the opposite transverse forces at the two Bloch-like regions of the kink, in contrast to the conventional skyrmion, the $1 \mathrm{kSk}$ would be subjected to a nonzero net transverse force, affecting its motion and orienting the kink.

If $\mathbf{J}_{\text {app }}=J_{\text {app }} \hat{\mathbf{x}}$, the DL-SOT force $\mathbf{F}_{\mathrm{DL}}$ can be computed according to the following expression [26]:

$$
F_{\mathrm{DL}, i}=\mu_{0} M_{s} t H_{\mathrm{DL}}^{0} \iint d x d y\left(m_{x} \frac{\partial m_{z}}{\partial x_{i}}-m_{z} \frac{\partial m_{x}}{\partial x_{i}}\right)
$$

where $H_{\mathrm{DL}}^{0}=C_{\mathrm{DL}} J_{\mathrm{app}} / \mu_{0}, C_{\mathrm{DL}}$ being the effective magnetic field per unit current density (in $\mathrm{T} \mathrm{A}^{-1} \mathrm{~m}^{2}$ ). By implementing the above equation for a $1 \mathrm{kSk}$ in case of horizontal current injection, the transverse force component $F_{\mathrm{DL}, y}$ was found to be seven orders of magnitude greater than for a Sk at $t=0 \mathrm{~ns}$. Also, the map of the DL-SOT force density revealed that $F_{\mathrm{DL}, y}$ is highly concentrated at the kink (not shown). This could ultimately explain the reorientation experienced by the DWSk during the transient time preceding the steady motion driven by SOT (see Supplemental Material Video SV2 [33]).

Also, we may notice in Fig. 4(f) that for current densities larger than $500 \times 10^{9} \mathrm{~A} / \mathrm{m}^{2}$, the curves for $1 \mathrm{kSk}$ merge with the one of the single skyrmion. This is because, in this high current density regime, the $1 \mathrm{kSk}$ changes its spin texture. This change depends on the angle of the injected current, as shown in Fig. 5 with snapshots of the transition: When the current orientation is the same as in Fig. 4(b), the $1 \mathrm{kSk}$ is transformed into a single skyrmion [Fig. 5(b), see Supplementary Video SV4]. In that case the polarization of the spin current $\sigma$ induced by the spin Hall effect in the heavy metal is aligned very close to the kink orientation axis. However, if the polarization of the spin current is rotated by $\pi / 2$, thus nearly perpendicular to the kink, the $1 \mathrm{kSk}$ is transformed into two single skyrmions. In the case of Fig. 5(b), the total topological charge is changed from $Q=2$ to $Q=1$, whereas in the case of Fig. 5(a) (see Supplementary Video SV3) and Fig. 5(c) (see Supplementary Video SV5) the total topological charge remains $Q=2$. Nevertheless, in these latter cases, the nature and dimensionality of the spin texture has been transformed 


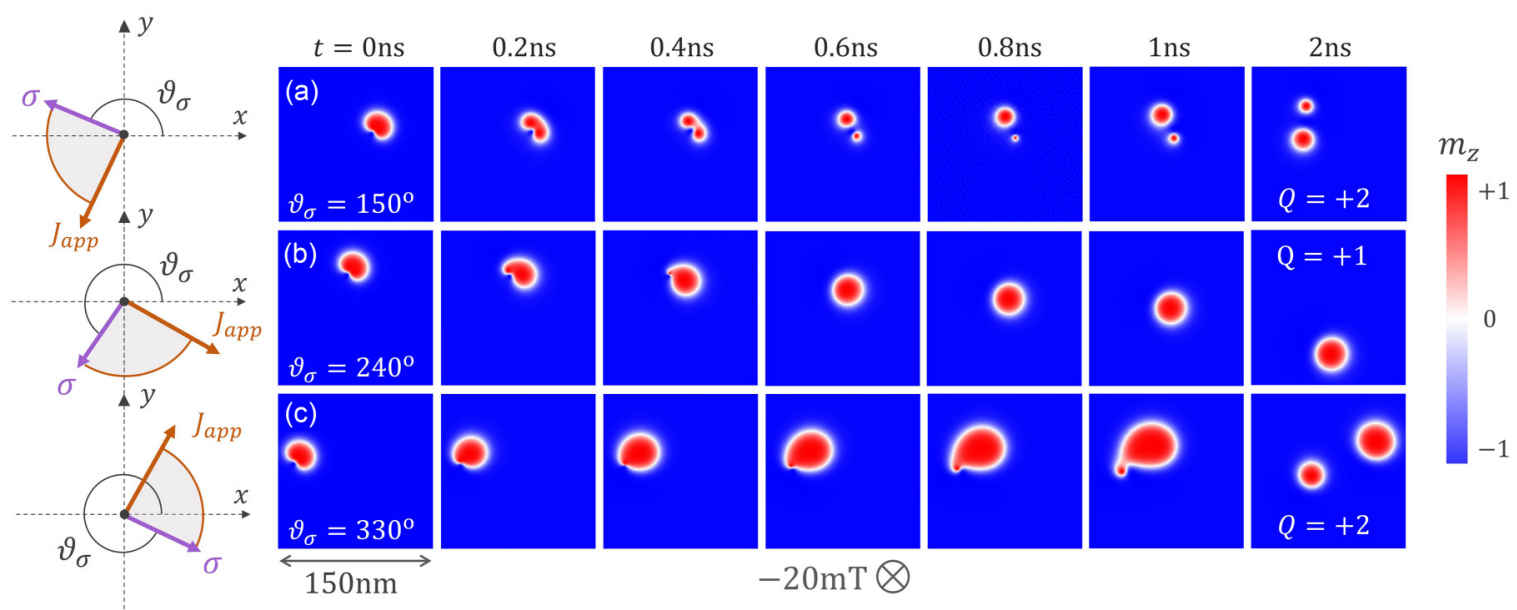

FIG. 5. High current SOT regime for various angles of the current with respect to the chiral kink if $J_{\text {app }}=100 \times 10^{10} \mathrm{~A} / \mathrm{m}^{2}$ and $\mu_{0} H_{\text {app }}=$ $-20 \mathrm{mT}$. The electrical current polarization is $10 \%$. The angle between the spin polarization $\sigma$ represented in the left column, perpendicular to the current direction due to the spin Hall effect, has been set either close to the kink orientation (b) or perpendicular to it (a), (c). The initial state at $0 \mathrm{~ns}$ is the same for the three cases, with a shift, so that the motion remains visible after 2 ns integration time. For a spin polarization $\sigma$ aligned with the kink, the 1-kink skyrmion transforms into a single skyrmion whereas for $\sigma$ perpendicular to the kink, it transforms in two single skyrmions.

between a single quasiparticle with double topological charge and two quasiparticles with a unitary topological charge. Such transformation might be interesting for applications as a 1kink skyrmion, with size very similar to a single skyrmion, could carry a higher density of topological charge that could be further separated into two single bits of information.

\section{1-KINK SKYRMION UNDER PERPENDICULAR STT}

The nonradial symmetry of a 1-kink skyrmion in a magnetic pillar is expected to bring additional features into the dynamics with respect to a conventional skyrmion. In particular, magnetization autooscillations, predicted for single skyrmion by spin-polarized injecting a current in nanopillar [34], could be used for spin-torque nano-oscillators (STNO) [35]. In order to check the $1 \mathrm{kSk}$ potential for this type of application, we integrated a $1 \mathrm{kSk}$ layer in a magnetic pillar. The layer containing the $1 \mathrm{kSk}$ is the free layer while the polarizer layer is perpendicularly magnetized [see inset of Fig. 6(a)]. We have simulated an injected current perpendicular to the layer plane, thus leading to STT with a spin polarization along $+z$ (see the details of the model in the Appendix).

We show in Fig. 6(c) that, upon injecting the current in the low/medium regime $\left(J_{\text {app }} \leqslant 200 \times 10^{10} \mathrm{~A} / \mathrm{m}^{2}\right)$, the $1 \mathrm{kSk}$ undergoes a rotation around the $z$ axis (see Supplementary Video SV6). When the current density is increased, the rotation frequency rises and the effective diameter is getting larger as well, as represented in Fig. 6(a). If we correct with the $1 \mathrm{kSk}$ size and plot frequency as a function of the current injected on the $1 \mathrm{kSk}$ surface, as represented in Fig. 6(b), we observe a first increase and a saturating behavior for currents above $\sim 10 \mathrm{~mA}$. The size increase is due to the polarizer orientation that favors the $1 \mathrm{kSk}$ core. As a consequence of the expansion of the $1 \mathrm{kSk}$ core size, the kink to domain wall lengths ratio drastically decreases, meaning that the impact of the kink, which propels the auto-oscillatory motion, becomes even less significant. In support of this claim we have observed (not shown) that, if the applied magnetic field is removed, the $1 \mathrm{kSk}$ core is enlarged, e.g., for $J_{\text {app }}=100 \times 10^{10} \mathrm{~A} / \mathrm{m}^{2}$, the frequency of rotation decreases by more than one order of magnitude.

The frequency reached for medium currents $\left(J_{\text {app }} \sim\right.$ $200 \times 10^{10} \mathrm{~A} / \mathrm{m}^{2}$ ) increases close to the $\mathrm{GHz}$ range. Intuitively, one can understand that reversal of current flux or, equivalently, of the spin polarizer (from up to down), could improve energy consumption. As an example, a current density of $J_{\text {app }}=100 \times 10^{10} \mathrm{~A} / \mathrm{m}^{2}$ (respectively $200 \times 10^{10} \mathrm{~A} / \mathrm{m}^{2}$ ), injected from the polarizer to the free layer under $\sim-20 \mathrm{mT}$, provides nearly the same rotational frequency $f \sim 118 \mathrm{MHz}$ (respectively $1 \mathrm{GHz}$ ) as of reversed STT current, but in the absence of any external magnetic field. Upon this, it is possible to infer that the concurrent action of negative STT current and Zeeman field may improve the effectiveness of the $1 \mathrm{kSk}$ auto-oscillation.

Figure 6(c) shows the $1 \mathrm{kSk}$ preferred sense of rotation, that is counterclockwise $(\mathrm{CCW})$ for $J_{\text {app }}>0$ and up polarizer. Upon switching the current sign, the sense of rotation is reversed as well. For the case of a DWSk localized within a flat domain wall, the computation of the dampinglike (DL) torque effective magnetic field $\left(H_{\text {eff,DL }} \propto \mathbf{m} \times \hat{\mathbf{z}}\right)$ predicts a left displacement of the kink. This is in perfect agreement with numerical observations (not shown). Upon converting the linear domain wall into a circular one, the kink, while displacing towards the left, is forced to follow the contour of the domain wall, which acts as a track that cannot be escaped. The linear one-way (left/right) displacement is thus converted into a rotational motion with well-defined sense of rotation $(\mathrm{CCW} / \mathrm{CW})$, controlled by the sign of the injected current (positive/negative).

After simulating the behavior of $1 \mathrm{kSk}$ under perpendicular STT with low/medium currents, we have increased the current density and observed that it could be an alternative approach to nucleate these spin textures with domain wall skyrmions. Indeed, by slightly reinforcing the STT current density above 

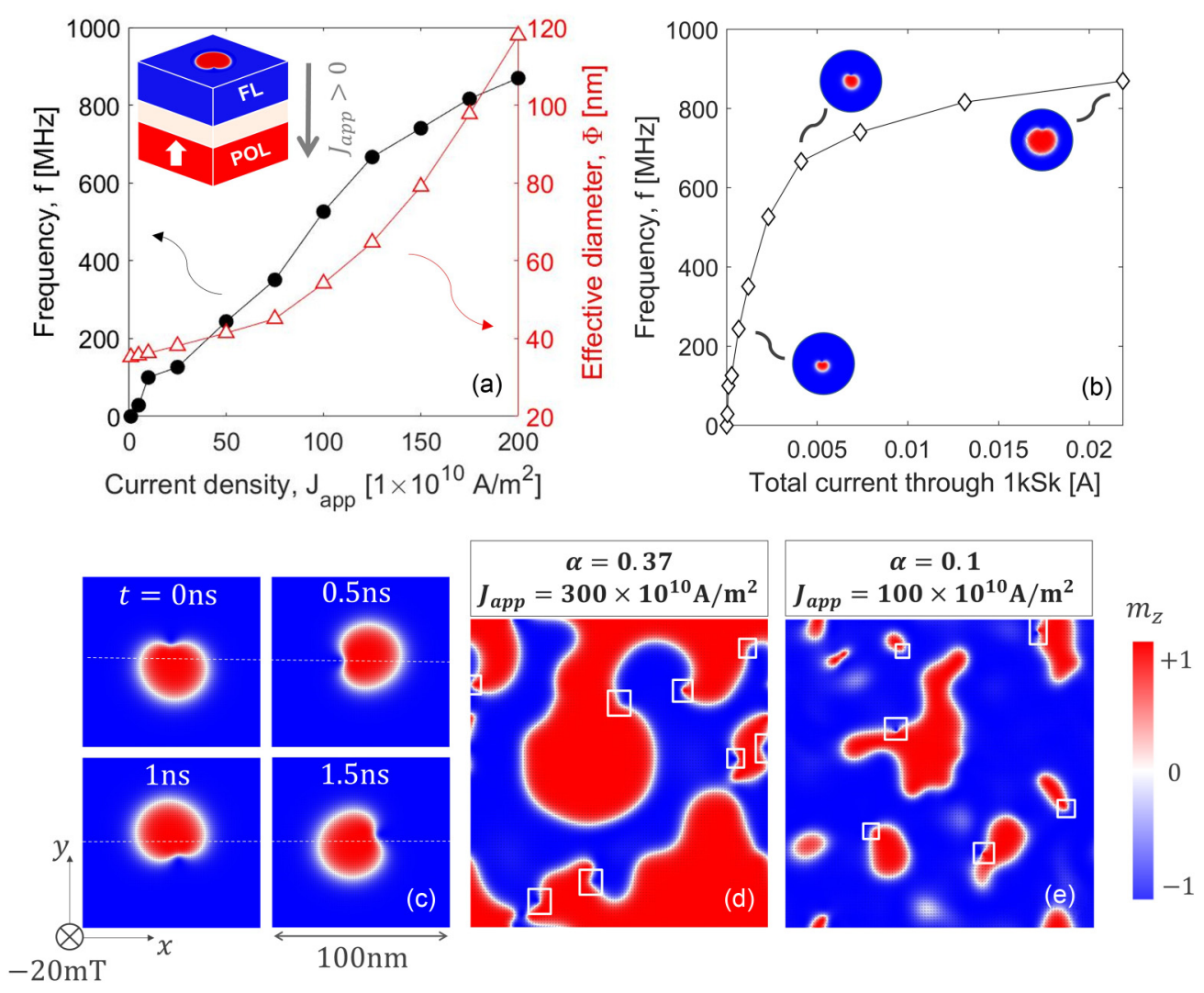

FIG. 6. STT driven dynamics with perpendicular polarizer and $10 \%$ of spin polarization. (a) Evolution of the frequency of oscillation (black curve) and effective diameter (red curve) vs current density. Inset: representation of the magnetic pillar with the bottom polarizer layer polarized along $+z$ and the top layer containing the 1-kink skyrmion, separated by a thin insulator. (b) Evolution of the frequency of oscillation vs total electrical current injected through the 1-kink skyrmion. (c) Snapshots of the 1-kink skyrmion orientation after $0.5 \mathrm{~ns}$ integration time steps for $J_{\text {app }}=100 \times 10^{10} \mathrm{~A} / \mathrm{m}^{2}$ and $\mu_{0} H_{\text {app }}=-20 \mathrm{mT}$. Snapshots of magnetic layer dynamic equilibrium configuration during integration in moderately high current regime for two different values of the damping parameter: (d) $J_{\text {app }}=300 \times 10^{10} \mathrm{~A} / \mathrm{m}^{2}, \alpha=0.37$ and (e) $J_{\text {app }}=100 \times 10^{10} \mathrm{~A} / \mathrm{m}^{2}, \alpha=0.1$. Domain wall skyrmions are highlighted through white squares.

a critical value (function of the damping parameter), without saturating the magnetic layer, the $1 \mathrm{kSk}$ is destroyed and different magnetic multidomains are nucleated. Interestingly, their dynamic evolution reveals the presence of several kinks and antikinks localized in the domain walls [see white squares in Figs. 6(d) and 6(e)]. This observation opens the perspective of using such pillar for both nucleating 1 or n-kink or antikink skyrmions and further activating their auto-oscillation.

After their nucleation, a last bottleneck for n-kink skyrmion use would be to observe them. For that, as discussed above, LTEM would be a method of choice. Alternatively, if resolution is not good enough to observe the internal structure of the kink, it should be possible to distinguish between a distortion due to defects and a chiral kink by injecting a current and observe their SOT induced motion and split into two single skyrmions.

\section{CONCLUSION}

To conclude, we have shown by micromagnetic simulations that in $\mathrm{Pt} / \mathrm{Co} / \mathrm{MgO}$ stack, a domain wall skyrmion may be stabilized within the Néel domain wall of a skyrmion, forming a 1-kink skyrmion. This was possible by refining the mesh beyond the usual mesh size. We have shown that these 1-kink skyrmions are stable versus anisotropy distri- bution as well as thermal fluctuation. Their behavior under magnetic field is similar to the one of conventional skyrmions and they are more robust than usual skyrmions to a decrease of Dzyaloshinskii-Moriya interaction, due to their larger topological charge. We have also shown that they can be manipulated by a current, similarly to usual skyrmions. Noticeable differences are (i) that the domain wall skyrmion breaks the radial symmetry of the spin texture and it orients itself under current, which is an additional degree of freedom for its manipulation, and (ii) that 1-kink skyrmion may be split into single skyrmions by using large enough SOT currents, making them interesting for high density information carrier. Finally, the auto-oscillations that 1-kink skyrmion show under a perpendicular STT current lead to tuneable frequency and make them interesting for STNO applications. However, since the internal structure of the 1-kink skyrmion implies a very steep magnetization gradient, its precise reaction to the transfer of angular momentum calls for an extended analyses based on self-consistent coupling between LLG and spindependent transport equations [36]. The joint influence of defects, thermal effects, and higher-order spin orbit-torque terms on the motion of the 1-kink skyrmion requires a detailed description of the sample and a relevant calibration of micromagnetic model against experimental parameters $[19,22]$. The 
establishment of optoelectrical control of such topological spin textures, i.e., by using ultrafast laser pulses, combined with spin-polarized current and gate voltage, would break new ground to design novel high-density skyrmionic devices.

\section{ACKNOWLEDGMENTS}

This work was supported by the French Agence Nationale de la Recherche ANR-19-CE24-0019-01 and Defense Advanced Research Projects Agency-Topological Excitations in Electronics program Grant No. MIPR HR0011831554. We thank Claire Baraduc and Laurent Ranno for fruitful discussions.

\section{APPENDIX}

\section{Thermal fluctuations}

The micromagnetic simulations presented in Secs. II and III use the thermal field defined in MuMax3 as a fluctuating field $\boldsymbol{B}_{\text {therm }}$ which randomly changes between time steps [17]:

$$
\boldsymbol{B}_{\text {therm }}=\eta(\text { step }) \sqrt{\frac{2 \mu_{0} \alpha k_{B} T}{B_{\text {sat }} \gamma_{L L} \Delta V \Delta T}}
$$

where $\alpha$ is the damping parameter, $k_{B}$ the Boltzmann constant, $T$ the user-defined temperature, $B_{\text {sat }}$ the saturation magnetization measured in $(\mathrm{T}), \gamma_{\mathrm{LL}}$ the gyromagnetic ratio measured in $\left(\mathrm{T}^{-1} \mathrm{~s}^{-1}\right), \Delta V$ the unit cell volume, $\Delta t$ the time step, and $\eta$ (step) a random vector with standard normal distribution evolving after every time step.

\section{In-plane current spin-transfer torque}

The mechanism of spin-transfer torque is modeled in $\mathrm{Mu}-$ Max3 according to Zhang and $\mathrm{Li}$, valid in case of in-the-plane transport in a track, i.e., when an unpolarized current enters a nonuniformly magnetized ferromagnet. In the LandauLifshitz-Gilbert (LLG) formalism it reads [17]:

$$
\begin{gathered}
\boldsymbol{\tau}_{Z L}=-\boldsymbol{m} \times(\boldsymbol{m} \times[b \boldsymbol{J} \cdot \nabla] \boldsymbol{m})-\beta^{\prime} \boldsymbol{m} \times[b \boldsymbol{J} \cdot \nabla] \boldsymbol{m} \\
b=\frac{P \mu_{B}}{e M_{S}\left(1+\beta^{\prime 2}\right)}
\end{gathered}
$$

where $\boldsymbol{J}$ is the current density, $\beta^{\prime}$ is the degree of nonadiabaticity, $P$ is the polarization of the spin-polarized current, $\mu_{B}$ the Bohr magneton, $e$ the electron charge, and $M_{s}$ the saturation magnetization. In our STT micromagnetic simulations from Sec. V, $\beta^{\prime}$ and $P$ were set to 0 and 1 , respectively. $J$ is named injected current $J_{\text {app }}$.

\section{Spin-orbit torque}

In case of current perpendicular to plane transport in a nanopillar composed of a fixed layer (spin polarizer), a spacer, and a free layer, MuMax3 provides a spin momentum torque term following Slonczewski and Berger which, in the LandauLifshitz (LL) formalism, reads [17]:

$$
\begin{aligned}
\tau_{S L} & =\beta \frac{\epsilon+\alpha \epsilon^{\prime}}{1+\alpha^{2}}\left(\boldsymbol{m} \times\left(\boldsymbol{m}_{p} \times \boldsymbol{m}\right)\right)-\beta \frac{\epsilon-\alpha \epsilon^{\prime}}{1+\alpha^{2}} \boldsymbol{m} \times \boldsymbol{m}_{p} \\
\beta & =\frac{J_{z} \hbar}{M_{s} e d} \\
\epsilon & =\frac{P(\boldsymbol{r}, t) \Lambda^{2}}{\left(\Lambda^{2}+1\right)+\left(\Lambda^{2}-1\right)\left(\boldsymbol{m} \cdot \boldsymbol{m}_{p}\right)}
\end{aligned}
$$

where $J_{z}$ denotes the electric current density along the $z$ axis, $d$ the free layer thickness, $m_{p}$ the electron polarization direction, $P$ the spin polarization, $\Lambda$ the Slonczewski parameter characterizing the spacer layer, and $\epsilon$ the secondary spin-torque parameter.

In MuMax3, the spin-orbit torque can be implemented by setting $\Lambda=1$ and $\epsilon^{\prime}=0$ [31]. In this context, $\boldsymbol{m}_{p}$ can be better denoted as $\sigma$, representing the interface spin polarization, and $J_{z} P$ as $J_{c} \theta_{\mathrm{SH}}$, denoting the transverse spin current, with $J_{c}$ the longitudinal charge current injected in the heavy-metal layer and $\theta_{\mathrm{SH}}$ the spin-Hall angle in the underlying heavy metal.

In our SOT micromagnetic simulations from Sec. V, $\Lambda, \epsilon^{\prime}$ and $P$ were set to 1,0 and 0.1 , respectively. Different in-plane $\boldsymbol{m}_{p}(\boldsymbol{\sigma})$ configurations were explored (Fig. 5). $J_{c}$ is named injected current $J_{\text {app }}$.

\section{Perpendicular-to-plane current spin-transfer torque}

In order to perform perpendicular STT micromagnetic simulations, the parameters $\Lambda, \epsilon^{\prime}$, and $P$ were set to 1,0 , and 0.1 , respectively. $\boldsymbol{m}_{p}$ was set to $(0,0,+1)$ to represent a perpendicular polarizer. $J_{z}$ is named injected current $J_{\text {app }}$ in our figures. The mechanism of SOT with out-of-plane interface spin polarization can be regarded as conceptually equivalent to STT with perpendicular spin polarizer.
[1] A. N. Bogdanov and D. A. Yablonskii, Thermodynamically stable vortices in magnetically ordered crystals: The mixed state of magnetics, Zh. Eksp. Teor. Fiz. 95, 178 (1989) [Sov. Phys. JETP 68, 101 (1989)].

[2] W. Jiang, P. Upadhyaya, W. Zhang, G. Yu, M. B. Jungfleisch, F. Y. Fradin, J. E. Pearson, Y. Tserkovnyak, K. L. Wang, O. Heinonen, S. G. E. te Velthuis, and A. Hoffmann, Blowing magnetic skyrmion bubbles, Science 349, 283 (2015).

[3] S. Woo, K. Litzius, B. Krüger, M.-Y. Im, L. Caretta, K. Richter, M. Mann, A. Krone, R. M. Reeve, M. Weigand, P. Agrawal, I. Lemesh, M.-A. Mawass, P. Fischer,
M. Kläui, and G. S. D. Beach, Observation of roomtemperature magnetic skyrmions and their current-driven dynamics in ultrathin metallic ferromagnets, Nat. Mater. 15, 501 (2016).

[4] Y. Huang, W. Kang, X. Zhang, Y. Zhou, and W. Zhao, Magnetic skyrmion-based synaptic devices, Nanotechnology 28, 08LT02 (2017).

[5] D. Pinna, F. Abreu Araujo, J.-V. Kim, V. Cros, D. Querlioz, P. Bessiere, J. Droulez, and J. Grollier, Skyrmion Gas Manipulation for Probabilistic Computing, Phys. Rev. Appl. 9, 064018 (2018). 
[6] M. A. Azam, D. Bhattacharya, D. Querlioz, and J. Atulasimha, Resonate and fire neuron with fixed magnetic skyrmions, J. Appl. Phys. 124, 152122 (2018).

[7] I. E. Dzyalosinskij, Thermodynamic theory of weak ferromagnetism in antiferromagnetic substances, Sov. Phys., JETP 5, 1259 (1957).

[8] I. Dzyaloshinsky, A thermodynamic theory of "weak" ferromagnetism of antiferromagnetics, J. Phys. Chem. Solids 4, 241 (1958).

[9] T. Moriya, Anisotropic superexchange interaction and weak ferromagnetism, Phys. Rev. 120, 91 (1960).

[10] H. Braun, Topological effects in nanomagnetism: from superparamagnetism to chiral quantum solitons, Adv. Phys. 61, 1 (2012).

[11] N. Nagaosa and Y. Tokura, Topological properties and dynamics of magnetic skyrmions, Nat. Nanotechnol. 8, 899 (2013).

[12] R. Cheng, M. Li, A. Sapkota, A. Rai, A. Pokhrel, T. Mewes, C. Mewes, D. Xiao, M. De Graef, and V. Sokalski, Magnetic domain wall skyrmions, Phys. Rev. B 99, 184412 (2019).

[13] M. Li, A. Rai, A. Pokhrel, A. Sapkota, C. Mewes, T. Mewes, D. Xiao, M. D. Graef, and V. Sokalski, Magnetic domain wall substructures in $\mathrm{Pt} / \mathrm{Co} / \mathrm{Ni} / \mathrm{Ir}$ multi-layers, J. Appl. Phys. 130, 153903 (2021).

[14] V. M. Kuchkin, B. Barton-Singer, F. N. Rybakov, S. Blügel, B. J. Schroers, and N. S. Kiselev, Magnetic skyrmions, chiral kinks, and holomorphic functions, Phys. Rev. B 102, 144422 (2020).

[15] S.-G. Je, Topological charge analysis of dynamic process of transition to néel-type skyrmion: Role of domain wall skyrmions, Curr. Appl. Phys. 21, 175 (2021).

[16] C. Zhang, J.-N. Wang, C.-K. Song, N. Mehmood, Z. Zeng, Y.-X. Ma, J.-B. Wang, and Q.-F. Liu, Edge-guided heart-shaped skyrmion, Rare Metals (2021).

[17] A. Vansteenkiste, J. Leliaert, M. Dvornik, M. Helsen, F. GarciaSanchez, and B. Van Waeyenberge, The design and verification of MuMax3, AIP Adv. 4, 107133 (2014).

[18] O. Boulle, J. Vogel, H. Yang, S. Pizzini, D. de Souza Chaves, A. Locatelli, T. O. Menteş, A. Sala, L. D. BudaPrejbeanu, O. Klein, M. Belmeguenai, Y. Roussigné, A. Stashkevich, S. M. Chérif, L. Aballe, M. Foerster, M. Chshiev, S. Auffret, I. M. Miron, and G. Gaudin, Room-temperature chiral magnetic skyrmions in ultrathin magnetic nanostructures, Nat. Nanotechnol. 11, 449 (2016).

[19] R. Juge, S.-G. Je, D. d. S. Chaves, L. D. Buda-Prejbeanu, J. Peña-Garcia, J. Nath, I. M. Miron, K. G. Rana, L. Aballe, M. Foerster, F. Genuzio, T. O. Menteş, A. Locatelli, F. Maccherozzi, S. S. Dhesi, M. Belmeguenai, Y. Roussigné, S. Auffret, S. Pizzini, G. Gaudin et al., Current-Driven Skyrmion Dynamics and Drive-Dependent Skyrmion Hall Effect in an Ultrathin Film, Phys. Rev. Appl. 12, 044007 (2019).

[20] S.-G. Je, P. Vallobra, T. Srivastava, J.-C. Rojas-Sánchez, T. H. Pham, M. Hehn, G. Malinowski, C. Baraduc, S. Auffret, G. Gaudin, S. Mangin, H. Béa, and O. Boulle, Creation of magnetic skyrmion bubble lattices by ultrafast laser in ultrathin films, Nano Lett. 18, 7362 (2018).

[21] F. Büttner, B. Pfau, M. Böttcher, M. Schneider, G. Mercurio, C. M. Günther, P. Hessing, C. Klose, A. Wittmann,
K. Gerlinger, L.-M. Kern, C. Strüber, C. von Korff Schmising, J. Fuchs, D. Engel, A. Churikova, S. Huang, D. Suzuki, I. Lemesh, M. Huang et al., Observation of fluctuation-mediated picosecond nucleation of a topological phase, Nat. Mater. 20, 30 (2021).

[22] R. Juge, S.-G. Je, D. de Souza Chaves, S. Pizzini, L. D. BudaPrejbeanu, L. Aballe, M. Foerster, A. Locatelli, T. O. Menteş, A. Sala, F. Maccherozzi, S. S. Dhesi, S. Auffret, E. Gautier, G. Gaudin, J. Vogel, and O. Boulle, Magnetic skyrmions in confined geometries: Effect of the magnetic field and the disorder, J. Magn. Magn. Mater. 455, 3 (2018).

[23] A. P. Malozemoff and J. C. Slonczewski, Effect of Bloch Lines on Magnetic Domain-Wall Mobility, Phys. Rev. Lett. 29, 952 (1972).

[24] J. C. Slonczewski, Theory of Bloch-Line and Bloch-Wall Motion, Journal of Applied Physics 45, 2705 (1974).

[25] A. Thiaville, J. Miltat, and J. B. Youssef, Dynamics of vertical bloch lines in bubble garnets: Experiments and theory, Eur. Phys. J. B 23, 37 (2001).

[26] A. Thiele, Steady-State Motion of Magnetic Domains, Phys. Rev. Lett. 30, 230 (1973).

[27] W. Jiang, X. Zhang, G. Yu, W. Zhang, X. Wang, M. B. Jungfleisch, J. E. Pearson, X. Cheng, O. Heinonen, K. L. Wang, Y. Zhou, A. Hoffmann, and S. te Velthuis, Direct observation of the skyrmion hall effect, Nat. Phys. 13, 162 (2017).

[28] K. Litzius, I. Lemesh, B. Krüger, P. Bassirian, L. Caretta, K. Richter, F. Büttner, K. Sato, O. A. Tretiakov, J. Förster, R. M. Reeve, M. Weigand, I. Bykova, H. Stoll, G. Schütz, G. S. D. Beach, and M. Kläui, Skyrmion hall effect revealed by direct time-resolved x-ray microscopy, Nat. Phys. 13, 170 (2017).

[29] J. Masell, D. R. Rodrigues, B. F. McKeever, and K. EverschorSitte, Magnetic skyrmions, chiral kinks, and holomorphic functions, Phys. Rev. B 101, 214428 (2020).

[30] N. Sisodia, P. K. Muduli, N. Papanicolaou, and S. Komineas, Chiral droplets and current-driven motion in ferromagnets, Phys. Rev. B 103, 024431 (2021).

[31] M. Dai and J. Hu, Field-free spin-orbit torque perpendicular magnetization switching in ultra-thin nanostructures, npj Comput. Mater. 6, 78 (2020).

[32] R. Tomasello, E. Martinez, R. Zivieri, L. Torres, M. Carpentieri, and G. Finocchio, A strategy for the design of skyrmion racetrack memories, Sci. Rep. 4, 6784 (2014).

[33] See Supplemental Material at http://link.aps.org/supplemental/ 10.1103/PhysRevB.104.174441 for the movies of the magnetization dynamics.

[34] N. Sisodia, S. Komineas, and P. K. Muduli, Chiral skyrmion auto-oscillations in a ferromagnet under spin-transfer torque, Phys. Rev. B 99, 184441 (2019).

[35] S. I. Kiselev, J. C. Sankey, I. N. Krivorotov, N. C. Emley, R. J. Schoelkopf, R. A. Buhrman, and D. C. Ralph, Microwave oscillations of a nanomagnet driven by a spin-polarized current, Nature (London) 425, 380 (2003).

[36] C. Petitjean, D. Luc, and X. Waintal, Unified Drift-Diffusion Theory for Transverse Spin Currents in Spin Valves, Domain Walls, and Other Textured Magnets, Phys. Rev. Lett. 109, 117204 (2012). 